\title{
Mitochondrial-related proteomic changes during obesity and fasting in mice are greater in the liver than skeletal muscles
}

\author{
Monika Nesteruk • Ewa E. Hennig • Michal Mikula • Jakub Karczmarski • Artur Dzwonek • \\ Krzysztof Goryca • Tymon Rubel • Agnieszka Paziewska • Marek Woszczynski • \\ Joanna Ledwon $\cdot$ Michalina Dabrowska $\cdot$ Michal Dadlez $\cdot$ Jerzy Ostrowski
}

Received: 10 April 2013 /Revised: 24 September 2013 / Accepted: 30 September 2013 /Published online: 1 November 2013

(C) The Author(s) 2013. This article is published with open access at Springerlink.com

\begin{abstract}
Although mitochondrial dysfunction is implicated in the pathogenesis of obesity, the molecular mechanisms underlying obesity-related metabolic abnormalities are not well established. We performed mitochondrial quantitative proteomic and whole transcriptome analysis followed by functional annotations within liver and skeletal muscles, using fasted and non-fasted 16- and 48-week-old high-fat diet (HFD)-fed and normal diet-fed (control group) wild-type C56BL/6J mice, and hyperphagic ob/ob and $\mathrm{db} / \mathrm{db}$ obese mice. Our study identified 1,675 and 704 mitochondriaassociated proteins with at least two peptides in liver and muscle, respectively. Of these, 221 liver and 44 muscle proteins were differentially expressed (adjusted $p$ values $\leq 0.05$ ) between control and all obese mice, while overnight fasting altered expression of 107 liver and 35 muscle proteins. In the liver, we distinguished a network of 27 proteins exhibiting
\end{abstract}

Electronic supplementary material The online version of this article (doi:10.1007/s10142-013-0342-3) contains supplementary material, which is available to authorized users.

M. Nesteruk · E. E. Hennig · A. Paziewska · J. Ledwon •

J. Ostrowski $(\bowtie)$

Department of Gastroenterology and Hepatology, Medical Center for Postgraduate Education, Roentgena 5, 02-781 Warsaw, Poland e-mail: jostrow@warman.com.pl

E. E. Hennig $\cdot$ M. Mikula $\cdot$ J. Karczmarski $\cdot$ A. Dzwonek $\cdot$

K. Goryca $\cdot$ M. Woszczynski $\cdot$ M. Dabrowska $\cdot$ J. Ostrowski Department of Genetics, Maria Sklodowska-Curie Memorial Cancer Center and Institute of Oncology, Warsaw, Poland

T. Rubel

Institute of Radioelectronics, Warsaw University of Technology, Warsaw, Poland

M. Dadlez

Institute of Biochemistry and Biophysics, Polish Academy of

Sciences, Warsaw, Poland opposite direction of expression changes in HFD-fed and hyperphagic mice when compared to control. The network centered on cytochromes P450 3a11 (Cyp3a11) and 4a14 (Cyp4a14), and fructose-bisphosphate aldolase B (Aldob) proteins which bridged proteins cluster involved in Metabolism of xenobiotics with proteins engaged in Fatty acid metabolism and PPAR signaling pathways. Functional annotations revealed that most of the hepatic molecular alterations, which characterized both obesity and fasting, related to different aspects of energy metabolism (such as Fatty acid metabolism, Peroxisome, and PPAR signaling); however, only a limited number of functional annotations could be selected from skeletal muscle data sets. Thus, our comprehensive molecular overview revealed that both obesity and fasting states induce more pronounced mitochondrial proteome changes in the liver than in the muscles.

Keywords Obesity $\cdot$ Mouse model $\cdot$ Mass spectrometry . Proteome $\cdot$ Mitochondria $\cdot$ Liver $\cdot$ Skeletal muscle

\section{Introduction}

Obesity is caused by an imbalance between energy intake and energy utilization. The excess of energy is accumulated in lipid droplets and prolonged caloric overconsumption results in a positive energy balance and increasing body weight. Several animal models were introduced to study behavioral and metabolic consequences of overnutrition (Larter and Yeh 2008). Of them, both prolonged feeding with a high-fat diet (HFD) and disruption of leptin signaling in leptin-deficient ob/ $\mathrm{ob}$ and leptin receptor-deficient $\mathrm{db} / \mathrm{db}$ hyperphagic mice were shown to result in obesity, insulin resistance, oxidative stress, changes in lipid and glucose homeostasis, and hepatic steatosis (Kennedy et al. 2010; Panchal and Brown 2011). However, since dietary behaviors significantly differ between 
mice prolonged fed with HFD and hyperphagic mice fed with normal diet (ND), they may exhibit differences also in molecular response to the deposition of a body fat.

Several microarray-based studies of obese humans and animal obesity models have been conducted during the past decade. Most of these studies have focused on identifying the transcriptional changes underlying the pathophysiology of obesity, as well as the anti-obesity effects of dietary, pharmacological, and behavioral interventions (Sun 2007). In a consequence, thousands of genes differentially expressed between lean and obese individuals have been identified, including those related to the hepatic glucose and lipid metabolism, insulin signaling, inflammation, coagulation, cell adhesion, oxygen stress, and activity of chaperone (Sharma et al. 2006; Greco et al. 2008; Kim 2010; Naik et al. 2013). On the contrary, a limited number of muscle transcriptomic studies have been reported so far. In one series of them, a shortterm feeding of mice with HFD changed the expression of more than 1,000 genes in the skeletal muscle (de Wilde et al. 2008), but an 8-week HFD feeding induced rather small alterations in the muscle transcriptome, with up-regulation of only Fatty acid oxidation pathway (de Wilde et al. 2009). Others studies revealed, however, that overall gene expression in muscle, involved in fatty acid oxidation and biosynthesis, was either decreased or obesity unresponsive (Kim 2010).

In contrast to microarray-based studies, only a few reports on the proteomic profiling in obesity can be found in the literature. In previous SDS-PAGE-based (Luo et al. 2012), 2D-based (Park et al. 2011; Eccleston et al. 2011), and 2D differential in-gel electrophoresis-based (Douette et al. 2005; Zhang et al. 2010; Kirpich et al. 2011; Thomas et al. 2012) analyses of total liver proteins from rodents chronically exposed to HFD, between 12 and 53 proteins reportedly displayed significant obesity-related changes in expression. After short-term feeding with HFD, one hepatic protein was significantly upregulated (Thomas et al. 2012). Recent quantitative proteomic analysis of total liver proteome identified 1,131 proteins and expression of 90 of them differed significantly between obese HFD-fed rats and lean rats (Baiges et al. 2010).

Because the ability of mass spectrometry (MS)-based approaches to identify proteins expressed at low levels may be enhanced when the analyzed peptides are restricted to selected sub-proteomes (Adachi et al. 2007; Altelaar and Heck 2012), we focused on mitochondrial (MT) proteome alterations. The MT sub-proteome is suitable for the detection of molecular alterations associated with obesity for several reasons: (1) obesity is a consequence of energy imbalance; (2) cellular energy is largely supplied by the MT oxidative phosphorylation system, which is regulated predominantly by insulin in muscle, liver, and adipose cells; (3) transcription rates from both MT and nuclear genomes are activated by insulin (Boirie 2003; Mikula et al. 2005), and genes that belong to MT metabolic, catabolic, and biosynthetic pathways are highly expressed in obese mice (Sharma et al. 2010); (4) MT dysfunctions have been implicated in the pathogenesis of obesity and obesity-associated diseases, including metabolic syndrome, non-alcoholic fatty liver disease, type 2 diabetes, and atherosclerotic heart disease (Samuel and Shulman 2012); and finally, (5) the mammalian MT proteome consists of slightly more than 1,000 proteins (Pagliarini et al. 2008).

Transcriptomic and proteomic results of the studies performed in obesity models have not been integrated so far. Furthermore, direct comparisons of liver and muscle MT proteomes in obesity models have not been performed to date. Here, MT proteomes were quantitatively evaluated using isobaric tags for relative and absolute quantitation (iTRAQ), followed by liquid chromatography-tandem MS (LC-MS/ MS) analysis, and transcript measurements were generated using microarray-based studies. Our studies reveal that combining MT proteomic and transcriptomic surveys enriches the functional analysis of obesity and fasting states in mice. Combined data on differentially expressed genes and proteins suggest that both obesity and fasting states result from highly disordered metabolic processes which in prolonged obesity is followed by deeper cellular and organismal systems deficiencies. In contrast, numbers of proteins and transcripts differentially expressed in muscle samples were too low to be assigned to any of functional pathways. Thus, altered expression of proteins and corresponding genes accompanied obesity and fasting states is quantitatively different in the liver and skeletal muscle.

\section{Materials and methods}

\section{Animals}

Ninety-six 5-week-old male, certificated mice were purchased from The Jackson Laboratory, USA. Of them, 48 mice were wild-type (wt) C57BL/6J, 24 mice were homozygous for the leptin gene mutation B6.V-Lep ${ }^{o b} / \mathrm{J}(\mathrm{ob} / \mathrm{ob})$, and 24 mice were homozygous for the leptin receptor gene mutation B6.BKS(D)-Lepr ${ }^{d b} / \mathrm{J}(\mathrm{db} / \mathrm{db})$. Throughout the study, mice were housed under temperature-controlled $\left(21 \pm 2{ }^{\circ} \mathrm{C}\right)$ and humidity-controlled $(55 \pm 10 \%)$ conditions, with a 12-h light/dark cycle and ad libitum access to food and water.

\section{Ethics statement}

Mice were housed in the Department of Genetics and Laboratory Animals Breeding, at the Maria Sklodowska-Curie Memorial Cancer Center and Institute of Oncology. Experimental protocols were approved by The 2nd Local Ethical Committee for Animal Research in Warsaw, Poland. 


\section{Experimental design}

Following purchase, mice were allowed 1-week adaptation, during which all animals were fed ND (10\% of calories from fat), containing $19.2 \%$ protein, $67.3 \%$ carbohydrate, and $4.3 \%$ fat (D12450B; Research Diets, New Brunswick, NJ, USA). At 6 weeks of age, 24 wt C57BL/6J mice were fed ND (control group) and other $24 \mathrm{wt} \mathrm{C57BL/6J} \mathrm{mice} \mathrm{were} \mathrm{fed} \mathrm{HFD}$ (HFD group) (60\% of calories from fat), containing $26.2 \%$ protein, $26.3 \%$ carbohydrate, and $34.9 \%$ fat (D12492; Research Diets). The ob/ob and db/db mice were fed ND throughout the entire experiment. To determine the effect of fasting, half of the mice in each group were deprived of food for a period of $18 \mathrm{~h}$ (between $3 \mathrm{PM}$ and $9 \mathrm{AM}$ next day) before sacrifice. An overview of the experimental design is shown in Fig. 1. At either 16 weeks or 48 weeks of age, mice were weighed and sacrificed, followed by rapid collection of blood, livers, and skeletal muscles. Fragments of fresh tissue samples used for mitochondria isolation were placed into ice-cold phosphate buffered saline (PBS). The remaining samples were snap-frozen and stored at $-72{ }^{\circ} \mathrm{C}$ until use.

\section{mRNA extraction}

Total RNA was isolated from liver and muscle samples using the RNeasy Plus Mini Kit (Qiagen, Germany) or TRIzol (Invitrogen Co., CA, USA), respectively, followed by oncolumn DNAse I digestion. The quality of RNA samples was determined using an Agilent 2100 Bioanalyzer (Agilent Technologies, CA, USA); samples used for microarray analysis displayed distinct peaks corresponding to intact $28 \mathrm{~S}$ and $18 \mathrm{~S}$ ribosomal RNA. Equal amounts of RNA (500 ng) were combined from two randomly selected animals. This process was performed for the six animals in each group, resulting in three biological replicates of pooled RNA per trait (Table S1 depicts the sample pooling process).

\section{Microarray analyses}

The average signal from MouseRef- 8 v2.0 Expression BeadChips (AROS Applied Biotechnology, Aarhus, Denmark) was quantile normalized with no background correction. All computations were performed using R 2.15.0 software (R Development Core Team 2011) with the Bioconductor extension (Gentleman et al. 2004). Illumina and Kyoto Encyclopedia of Genes and Genomes (KEGG) (Kanehisa and Goto 2000) identifiers were mapped to genes using lumiMouseAll.db (version 1.18.0), KEGG.db (version 2.8.0), and lumi (version 2.8.0) (Du et al. 2008) packages. Measurements were filtered according to the ratio of the interquartile range to the median. Only probes with a ratio greater than 1:8 were selected for analysis.

In the further analysis, only the probe sets corresponding to genes with detected proteins were considered in this study. Differentially expressed genes were identified by a $t$ test (Welch variant) followed by the Benjamini-Hochberg $p$ value correction for multiple hypotheses testing. Adjusted $p$ values $\leq 0.05$ were considered significant. Microarray data was deposited in Gene Expression Omnibus dataset GSE43691.

\section{Functional analyses}

The significance of mRNA and protein expression changes related to each KEGG pathway was assessed with the Kolmogorov-Smirnov (K-S) test. Resulting $p$ values were adjusted for multiple hypotheses testing with the Benjamini-Hochberg algorithm and values $\leq 0.05$ were considered significant.
Fig. 1 Overview of the experimental design. At the experiment endpoints, total RNA and mitochondrial protein extracts were combined from two animals (depicted as differential mice outlines) from each group of six, generating three biological replicate samples of pooled RNA and mitochondrial proteins per trait

\section{Start}

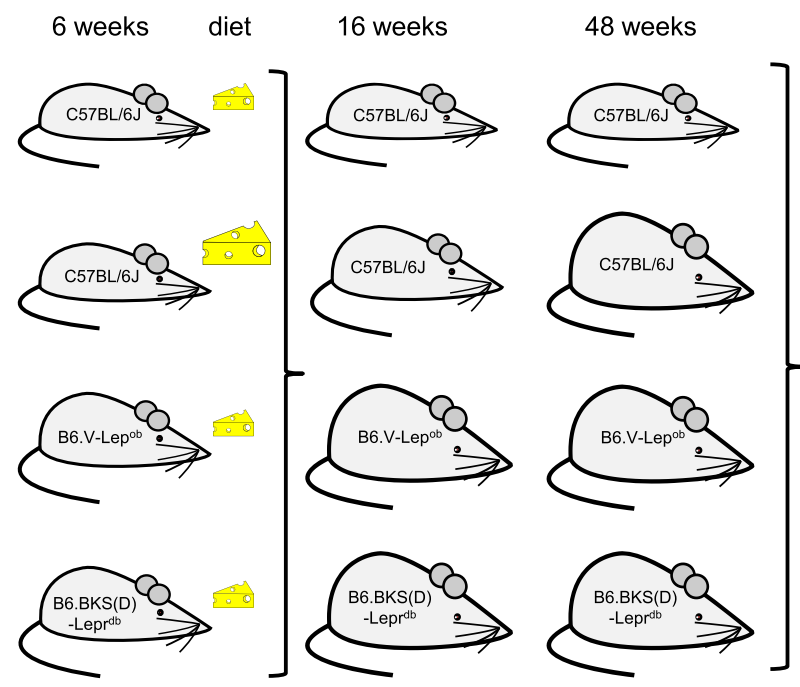

\section{Experimental flow}

12 mice per group

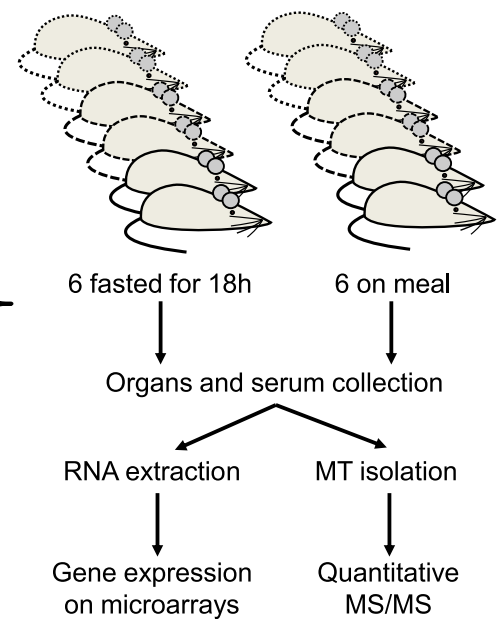


To explore the network of proteins and their contribution to KEGG pathways, we used the STRING software (Szklarczyk et al. 2011) that constructs functional protein-interaction networks and calculates its significance based on aggregated experimental data.

\section{Isolation of mitochondria}

Mitochondria from $100 \mathrm{mg}$ of mouse liver or hind limb muscle tissues were isolated and purified using differential centrifugation followed by ultracentrifugation in a discontinuous sucrose gradient (Ostrowski et al. 2004). The tissues were minced into small pieces using scissors and washed with ice-cold PBS. Minced muscles were incubated for $1 \mathrm{~h}$ in icecold PBS supplemented with $10 \mathrm{mM}$ EDTA and $0.05 \%$ trypsin to facilitate the homogenization process. Tissues were homogenized using a Mitochondria/Cytosol Fractionation Kit (BioVision, Milpitas, CA, USA). In brief, samples were homogenized in Cytosol Extraction Buffer supplemented with protease inhibitor cocktail, DTT, and phosphatase inhibitor cocktail 1 and 2 (Sigma). The homogenates were centrifuged (once for muscle, twice for liver) for $10 \mathrm{~min}$ at $740 \times g$ and $4{ }^{\circ} \mathrm{C}$, and then the unbroken tissue, cells, and nuclei were discarded. The supernatants were then centrifuged for $30 \mathrm{~min}$ at 10 , $000 \times g$ and $4{ }^{\circ} \mathrm{C}$ to pellet the mitochondria. MT pellets were suspended in ice-cold $0.25 \mathrm{M}$ sucrose, layered on $1 \mathrm{M}$ and $1.7 \mathrm{M}$ discontinuous sucrose gradients spiked with EDTA-free Complete Protease Inhibitor Cocktail (Roche, Basel, Switzerland), and then centrifuged for $45 \mathrm{~min}$ at $70,000 \times \mathrm{g}$ and $4{ }^{\circ} \mathrm{C}$. Mitochondria recovered from the gradient were diluted in ice-cold PBS and centrifuged for $20 \mathrm{~min}$ at $10,000 \times \mathrm{g}$ and $4{ }^{\circ} \mathrm{C}$. The purified mitochondria were stored at $-80{ }^{\circ} \mathrm{C}$.

\section{Protein extraction}

MT proteins were extracted with $8 \mathrm{M}$ urea in $2 \%$ CHAPS and concentrations were measured using a standard BCA assay. Equal amounts of MT protein $(100 \mu \mathrm{g})$ were combined from two randomly selected animals; this process was performed for the six animals in each group, resulting in three biological replicates, similarly as for microarray analyses (Table S1). Pooled samples were precipitated using the ProteoExtract Protein Precipitation Kit (Calbiochem, Germany), according to the manufacturer's protocol. Protein pellets were suspended in $40 \mu \mathrm{l}$ dissolution buffer $(0.5 \mathrm{M}$ triethylammonium bicarbonate, $0.1 \%$ SDS). To facilitate protein solubilization, samples were disrupted using a Bioruptor ultrasonic homogenizer (Diagenode, Belgium), using 10-s on-off cycles for 5 min at high intensity, and were then digested with trypsin at $37{ }^{\circ} \mathrm{C}$ overnight.

\section{iTRAQ labeling}

The concentration of tryptic peptides was measured using a modified BCA method (Kapoor et al. 2009). Equal amounts of peptides $(100 \mu \mathrm{g})$ were then reduced and cysteine-blocked according to the iTRAQ protocol. Peptides derived from a corresponding set of samples, comprising eight experimental traits per age group, were labeled using the iTRAQ 8plex kit (ABsciex, Framingham, MA, USA), as shown in Table S1. Peptides were then mixed and fractionated simultaneously, using isoelectric focusing (IEF) on $24 \mathrm{~cm}$ IPG strips (pH 3.5 to 4.5; GE Healthcare), as described previously (Mikula et al. 2011). Strips were cut into 14 sections and peptides were extracted and stored at $-72{ }^{\circ} \mathrm{C}$. For each age group, three strips were analyzed; each strip represented three biological replicates (each replicate composed of MT proteomes derived from two different, randomly selected animals).

\section{LC-MS/MS}

Analysis was performed using an Orbitrap MS (Thermo Scientific, USA) coupled with a nanoAcquity LC system (Waters, USA). Spectrometer parameters were as follows: capillary voltage, $2.5 \mathrm{kV}$; cone, $40 \mathrm{~V} ; \mathrm{N}_{2}$ gas flow, 0 ; and $\mathrm{m} / \mathrm{z}$ range, 300-2,000. Samples were loaded from the autosampler tray (cooled to $10{ }^{\circ} \mathrm{C}$ ) to the precolumn [Symmetry $\mathrm{C} 18$, $180 \mu \mathrm{m} \times 20 \mathrm{~mm}, 5 \mu \mathrm{m}$ (Waters)] using a mobile phase of $100 \%$ MilliQ water acidified with $0.1 \%$ formic acid. Peptides were transferred to the nano-UPLC column [BEH130 C18, $75 \mu \mathrm{m} \times 250 \mathrm{~mm}, 1.7 \mathrm{~mm}$ (Waters)] by a gradient of $5-30 \%$ acetonitrile in $0.1 \%$ formic acid over $180 \mathrm{~min}$ at a flow rate of $250 \mathrm{nl} / \mathrm{min}$ and then directly eluted to the ion source of MS. A blank run was performed before each analysis to ensure the lack of cross-contamination from previous samples.

\section{LC-MS/MS data processing and analysis}

All MS/MS data were analyzed using Mascot (version 2.3.02; Matrix Science), which was configured to search the mouse Swiss-Prot protein sequence database and reversed versions of these sequences assuming semiTrypsin digestion. The database search was performed with a fragment ion mass tolerance of $0.8 \mathrm{Da}$ and a peptide mass tolerance of $40 \mathrm{ppm}$. ITRAQ8PLEX (N-TERM), ITRAQ8PLEX (K), and METHYLTHIO (C) were specified in Mascot as fixed modifications; ITRAQ8PLEX (Y) and OXIDATION (M) were specified as variable modifications. The statistical significance of peptide identifications was assessed by a target/decoy database search strategy, as described previously (Mikula et al. 2011). The quantitative iTRAQ analysis was performed using a set of iTRAQ-labeled unique peptides, which were unambiguously assigned to a single protein, with an estimated false 
discovery ratio (FDR) of 0.01 . The intensities of the iTRAQ reporter ion peaks were corrected for isotope impurity and then median-normalized to remove systematic bias. For the statistical analysis of protein ratios, a permutation-based test implemented in the Diffprot software was used (Malinowska et al. 2012). Protein ratios with corrected $p$ values $\leq 0.05$ in pair-wise comparisons were considered significant. To assign proteins intracellular localization, both Swiss-Prot and Gene Ontology (GO) (Ashburner et al. 2000) were used.

\section{Results}

In order to define molecular alteration associated with obesity and fasting states in young and old obese mice, we used hepatic and muscle samples from mice at 16 and 48 weeks of age. Ten weeks of HFD feeding (16 weeks of mice age) was chosen since at this time HFD-fed mice already show obesity phenotypes, while some of 48-week-old obese mice started to show enhanced symptoms of severe disease, including failure to drink and eat, slower respiration, and impaired responsiveness to touch. A total of 12 mice per group (given genotype, diet, and age) were analyzed. Six mice in each group were deprived of food for a period of $18 \mathrm{~h}$ before sacrifice, according to typical mice metabolic studies, when an overnight fasting (for 14-18 h) has been usually employed (Ayala et al. 2010). Three biological replicates per group were generated by combining an equal amount of total RNA or MT protein extracts from two randomly selected animals (Fig. 1).

As expected, the body weights of both 16-week-old and 48-week-old ob/ob, db/db, and HFD-fed wt mice (determined immediately prior to sacrifice) were significantly higher than the weights of age-matched ND-fed control mice (Fig. 2a).
Moreover, ob/ob and $\mathrm{db} / \mathrm{db}$ mice at 16 weeks of age developed hyperinsulinemia, hyperglycemia, and moderate to severe hepatic steatosis, and 48 -week-old ob/ob, $\mathrm{db} / \mathrm{db}$ as well as wt $\mathrm{C} 57 \mathrm{BL} / 6 \mathrm{~J}$ mice (in parallel with prolonged HFD feeding) showed severe steatosis with moderate inflammation (our yet unpublished observations). Compared with the nonfasting condition, fasting for $18 \mathrm{~h}$ resulted in significantly reduced liver weight in young ob/ob and HFD-fed mice, as well as in both young and old control mice (Fig. 2b). Additionally, overnight fasting resulted in significantly reduced serum glucose concentration in young and old control mice, as well as in young HFD-fed mice, and reduced serum insulin concentration in all studied mice (not shown). Although both the glucose and insulin concentrations were significantly affected by the obese state, we could not diagnose diabetes in any of the obese groups (not shown) according to its accepted diagnostic criteria for mice (Andrikopoulos et al. 2008).

\section{Comparison of liver and muscle proteomes}

Isolated mitochondria were purified and the extracted peptides were labeled with iTRAQ, fractionated with IEF, and analyzed by LC-MS/MS. A total of 168 analytical LC-MS/MS runs were performed, producing 2,566,757 MS/MS spectra. A mouse Swiss-Prot protein sequence database search identified 17,606 and 10,967 peptides (estimated FDR $\leq 0.01$ ) that were assigned to 2,746/1,675 and 1,484/704 proteins (identified by at least one/two peptides) in liver and muscle samples, respectively, which represented both MT and co-purified non-MT proteomes (Table S2). Of these proteins, 904/490 were present in data sets from both tissue types. MT proteins were then selected using information deposited in the Swiss-Prot and GO databases. According to the Swiss-Prot localization information, 513/406 hepatic and 504/396 muscle MT proteins a

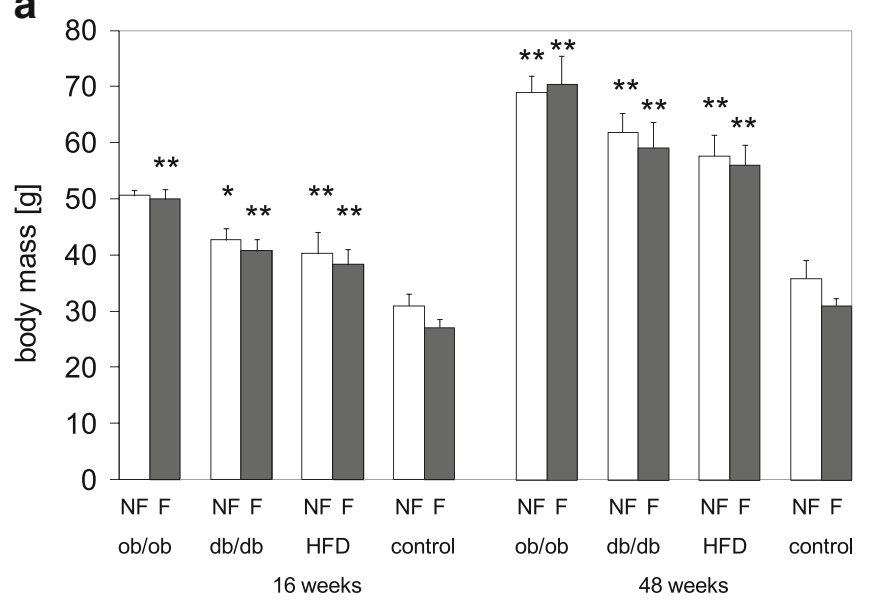

b

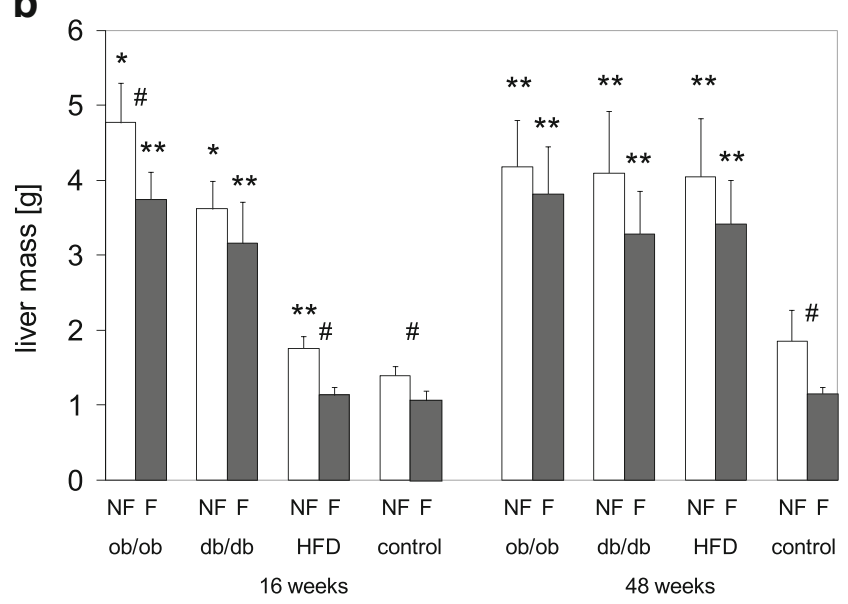

Fig. 2 Mouse total body weight (a) and liver weight (b) according to genotype and diet. $H F D$ fed a high-fat diet, $N F$ non-fasted, $F$ fasted. $* p<0.01$, $* * p<0.005$, compared with the relevant control group; $\# p<0.01$ for liver weight comparison between non-fasted and fasted mice in a given group 
were identified by one/two peptides. According to the GO term "mitochondria" (GO:0005739), 729/565 hepatic MT proteins and 504/396 muscle MT proteins were identified by one/two peptides. For clarity of further quantitative analyses, we considered only proteins identified by at least two peptides.

Proteins with an MT origin status in the Swiss-Prot database represented $22 \%$ and $53 \%$ of all proteins isolated from liver and muscle MT samples, respectively (Fig. 3). By comparison, MT proteins assigned by GO annotation represented $34 \%$ of all hepatic proteins and $56 \%$ of all muscle proteins identified. Notably, the hnRNP $\mathrm{K}$ protein, which was shown to be localized in mitochondria (Mikula et al. 2006), could not be attributed as a MT protein according to either the Swiss-Prot or GO data sets. Additionally, a recently published study on MT proteome mapping in living cells identified over 30 proteins within the human MT matrix that were not previously linked to mitochondria (Rhee et al. 2013). Since MT proteome is still not well established, for further analyses we used data for all proteins isolated with MT fractions.

To compare the protein abundances with the corresponding mRNA transcript levels, we performed microarray measurements of pooled RNA samples combined from same two animals as MT protein pools (Table S1). In total, 48 microarrays were hybridized with liver samples and 48 additional microarrays were hybridized with muscle samples.

Molecular alterations underlying obesity induced by hyperphagia and HFD

Obesity-related differentially expressed proteins (both MT and co-purified non-MT) were analyzed in pair-wise comparisons of non-fasted mice; the analysis was independent for each group of obese mice. The analysis of liver samples identified 123,115 , and 67 proteins that distinguished (adjusted $p$ values $\leq 0.05$ ) young control mice from young ob/ob, $\mathrm{db} /$ $\mathrm{db}$, and HFD-fed mice, respectively, and also 74, 79, and 41 proteins that distinguished old control mice from old ob/ob, $\mathrm{db} / \mathrm{db}$, and HFD-fed mice, respectively (Table 1). Analysis of muscle samples revealed 19, 22, and 7 proteins that distinguished young control mice from young $\mathrm{ob} / \mathrm{ob}, \mathrm{db} / \mathrm{db}$, and HFD-fed mice, respectively, and 5, 12, and 13 proteins that distinguished old control mice from old ob/ob, db/db, and HFD-fed mice, respectively (Table 1). The Venn diagrams on Fig. 4 present the number of differentially expressed proteins, common or unique between models of obesity. In sum, 221 proteins were significantly altered (adjusted $p$ values $\leq$ 0.05 ) in liver (Table S3), and 44 proteins were altered in muscle (Table S4).

Interestingly, a set of 27 hepatic proteins exhibited opposite direction of expression changes in HFD-fed and hyperphagic mice when compared to control (Table 2). Of them, 22 proteins appeared as differential in 16-week-old animals. Molecular context of these proteins interaction was next explored with STRING database. Analysis revealed that these proteins are highly interconnected (protein-protein interactions $p$ value 5.66E-15) (Fig. 5) and significantly enriched for KEGG fatty acids-related pathways (Peroxisome, PPAR signaling, Fatty acid metabolism) and xenobiotics metabolism. The central proteins of network are cytochrome P450 3a11 (Cyp3a11), fructose-bisphosphate aldolase B (Aldob), and Cyp4a14 which bridged proteins cluster involved in Metabolism of xenobiotics with components of Fatty acid metabolism and PPAR signaling pathways. According to STRING, the best documented associations in the network are between Cyp3a11 and Cyp4a14 proteins. Eight of these 27 proteins
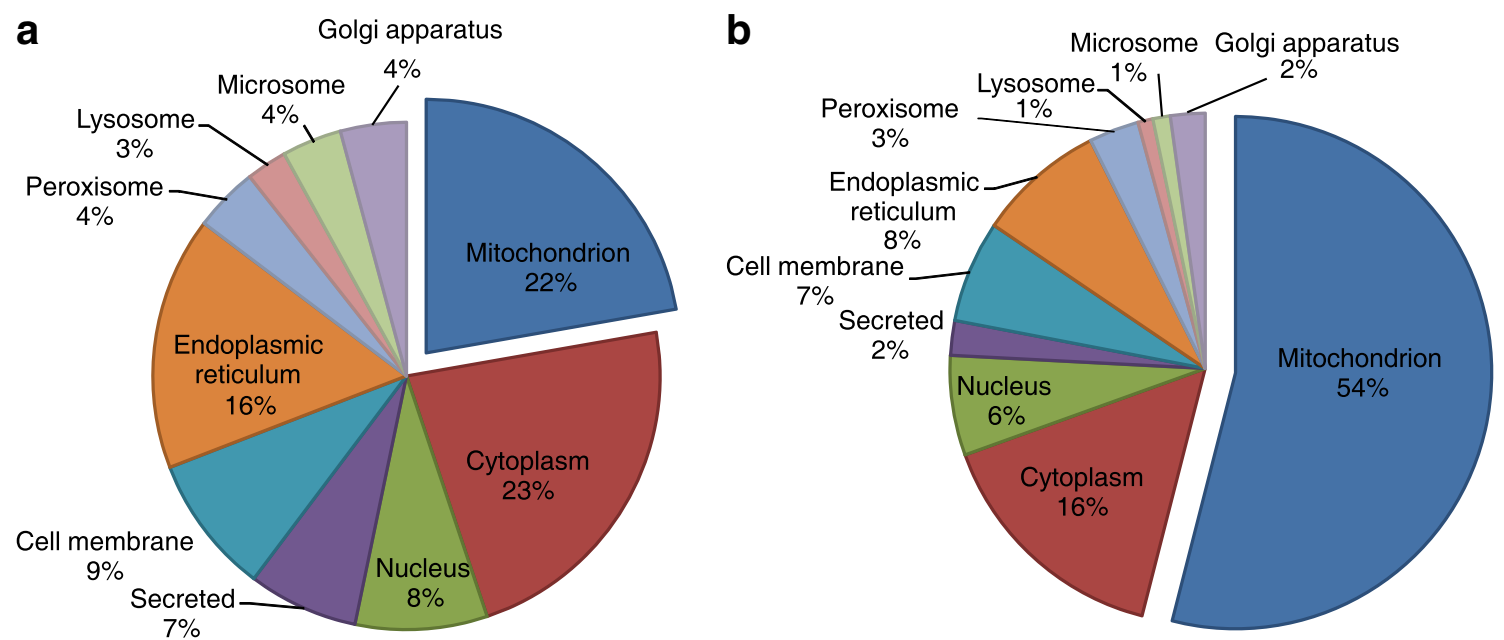

Fig. 3 Subcellular identity of the 1,675 and 704 proteins identified by at least two peptides in mitochondrial fractions isolated from liver (a) and muscle (b), respectively. Proteins were identified using the Swiss-Prot database 
Table 1 Numbers of detected proteins and numbers of proteins that corresponded to measurable mRNA used for comparisons between obese mice and appropriate control mice

\begin{tabular}{|c|c|c|c|c|c|c|}
\hline & \multicolumn{3}{|l|}{16 weeks } & \multicolumn{3}{|l|}{48 weeks } \\
\hline & $\mathrm{ob} / \mathrm{ob}$ & $\mathrm{db} / \mathrm{db}$ & HFD & $\mathrm{ob} / \mathrm{ob}$ & $\mathrm{db} / \mathrm{db}$ & HFD \\
\hline \multicolumn{7}{|l|}{ Hepatic proteins } \\
\hline $\begin{array}{l}\text { Total analyzed proteins (proteins with corresponding } \\
\text { probe sets in filtered dataset) }\end{array}$ & $1,242(948)$ & $1,250(951)$ & $1,260(960)$ & $1,077(828)$ & $1,080(826)$ & $1,067(820)$ \\
\hline Differentially expressed proteins & 123 & 115 & 67 & 74 & 79 & 41 \\
\hline \multicolumn{7}{|l|}{ Skeletal muscle proteins } \\
\hline $\begin{array}{l}\text { Total analyzed proteins (proteins with corresponding } \\
\text { probe sets in filtered dataset) }\end{array}$ & $546(419)$ & $548(421)$ & $547(420)$ & $459(353)$ & $457(351)$ & $459(353)$ \\
\hline Differentially expressed proteins & 19 & 22 & 7 & 5 & 12 & 13 \\
\hline
\end{tabular}

showed over 3-fold difference in expression between hyperphagic and HFD-fed mice at any of age point; four proteins were upregulated [glutathione $S$-transferase Mu 1 (Gstm1), ATP-binding cassette sub-family D member 2 (Abcd2), acylCoA desaturase 1 (Scd1), and fatty acid synthase (Fasn)] and four proteins were downregulated [cytochrome P450 4V3 (Cyp4V3), liver carboxylesterase 31 (Es31), ornithine aminotransferase (Oat), and serine-pyruvate aminotransferase (Agxt)] in hyperphagic mice. There were no proteins detected in the muscles that would have opposite expression in HFDfed and hyperphagic mice.
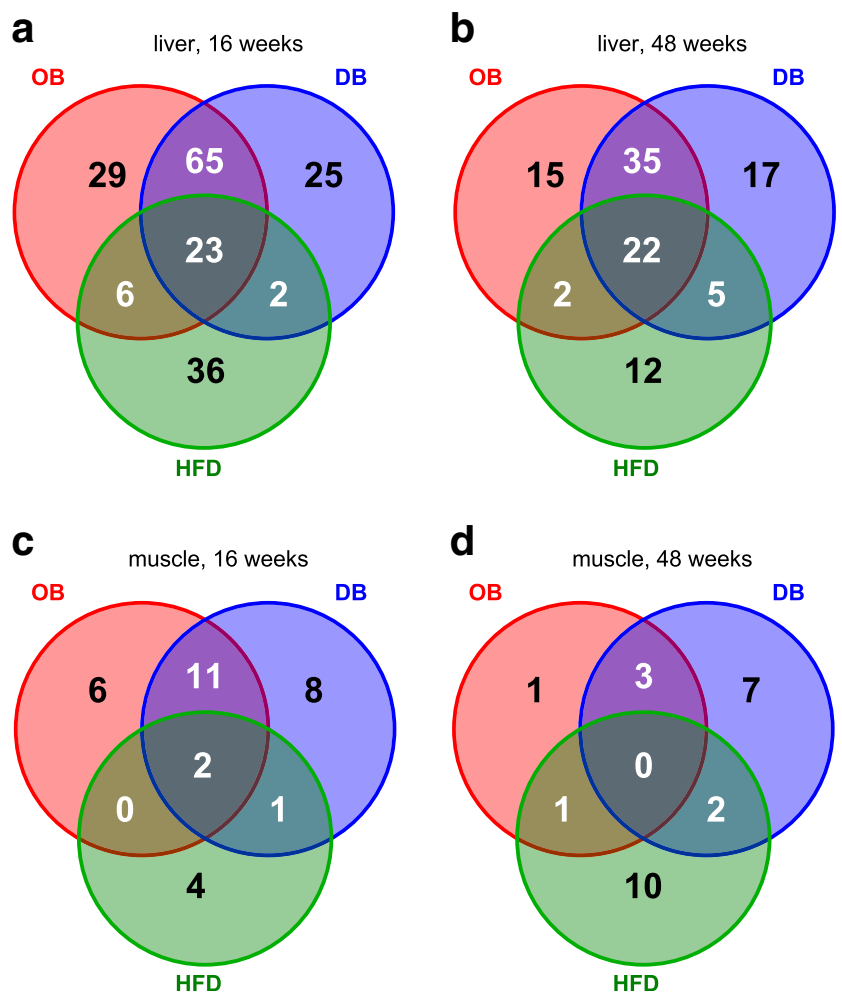

Fig. 4 Venn diagrams presenting common and unique differentially expressed proteins in different mice models of obesity
Next, all identified proteins and their corresponding probe sets, from liver or muscle samples (Table S5 and S6, respectively), were analyzed separately by annotation to pre-defined KEGG pathways (Table S7). Liver proteins could be assigned to 25 KEGG pathways in at least one comparison, while their corresponding probe sets were assigned to 18 pathways; of these, the Fatty acid metabolism, Biosynthesis of unsaturated fatty acids, Glycolysis/Gluconeogenesis, PPAR signaling, and Peroxisome pathways were common for the annotations of both proteins and their corresponding probe sets. Contrary to liver samples, no functional processes were selected from either muscle RNA or muscle protein obesity data sets.

\section{Molecular alterations underlying prolonged fasting}

Overnight fasting-related differentially expressed proteins were also independently identified in liver and muscle samples from each group of animals. In sum, fasting altered the expression (adjusted $p$ values $\leq 0.05$ ) of 107 proteins in liver samples and 35 proteins in muscle samples (Tables S8 and S9). The higher number of altered proteins was shown in both young and old control mice, comparing with all obese mice (Table 3). Noteworthy, a set of 45 hepatic proteins demonstrated concordant abundance change in response to fasting, in at least two groups of animals (Table S8); 38 of them were upregulated. When analyzed against the STRING database, these 45 proteins constructed a network significantly enriched in interactions that contribute to KEGG pathways related to fatty acids (Peroxisome, PPAR signaling, Fatty acid metabolism) and amino acids metabolism (Fig. 6). Four and three of differentially expressed proteins were common for all groups of young or old mice, respectively (Fig. 7a, b), while two of them, encoding components of cytochrome P450 (Cyp4a14 and Cyp4a10), were changed concordantly in both age groups (Table S8). 
Table 2 Differentially regulated proteins between hyperphagic and HFD-fed mice

\begin{tabular}{|c|c|c|c|c|c|c|c|c|}
\hline \multirow[t]{2}{*}{ Sp. acc. } & \multirow[t]{2}{*}{ Name } & \multirow[t]{2}{*}{ Gene } & \multicolumn{3}{|c|}{16 weeks } & \multicolumn{3}{|c|}{48 weeks } \\
\hline & & & $\mathrm{ob} / \mathrm{ob}$ & $\mathrm{db} / \mathrm{db}$ & HFD & $\mathrm{ob} / \mathrm{ob}$ & $\mathrm{db} / \mathrm{db}$ & HFD \\
\hline Q61285 & ATP-binding cassette sub-family D member 2 & Abcd2 & 3.44 & 2.95 & 0.59 & 1.72 & 1.65 & \\
\hline P10649 & Glutathione $S$-transferase $\mathrm{Mu} 1$ & Gstm1 & 2.05 & 2.64 & 0.58 & 1.82 & 1.55 & \\
\hline P13516 & Acyl-CoA desaturase 1 & Scd1 & 1.73 & & 0.53 & & & \\
\hline P19096 & Fatty acid synthase & Fasn & 1.65 & 1.64 & 0.58 & 1.83 & 1.56 & 0.57 \\
\hline Q64459 & Cytochrome P450 3A11 & Cyp3a11 & 1.35 & 1.51 & 0.66 & 1.50 & 1.18 & \\
\hline Q91Y97 & Fructose-bisphosphate aldolase B & Aldob & 1.89 & 1.62 & 0.74 & 2.12 & 1.50 & \\
\hline P17182 & Alpha-enolase & Eno1 & 1.69 & 1.40 & 0.80 & 1.74 & 1.54 & \\
\hline Q64521 & Glycerol-3-phosphate dehydrogenase, mitochondrial & Gpd2 & 1.64 & 1.61 & 0.87 & 1.60 & 1.50 & 1.43 \\
\hline O35728 & Cytochrome P450 4A14 & Cyp4a14 & 2.12 & 2.04 & 0.76 & & & 0.47 \\
\hline Q9DBM2 & Peroxisomal bifunctional enzyme & Ehhadh & 1.75 & 1.84 & 0.81 & & & 0.68 \\
\hline Q60597 & 2-Oxoglutarate dehydrogenase, mitochondrial & Ogdh & 1.41 & & 0.78 & & & \\
\hline P25688 & Uricase & Uox & 1.26 & & 0.79 & & & 0.88 \\
\hline P07724 & Serum albumin & Alb & & 1.36 & 0.83 & & & \\
\hline Q8VDN2 & Sodium/potassium-transporting ATPase subunit alpha- 1 & Atp1a1 & & & 0.84 & 1.50 & 1.27 & \\
\hline O35423 & Serine-pyruvate aminotransferase, mitochondrial & Agxt & 0.43 & & 1.96 & 0.62 & 0.53 & \\
\hline Q63880 & Liver carboxylesterase 31 & Es31 & 0.38 & 0.28 & 1.22 & 0.32 & 0.60 & \\
\hline P29758 & Ornithine aminotransferase, mitochondrial & Oat & 0.38 & 0.47 & 1.58 & 0.58 & 0.46 & 0.71 \\
\hline Q9DBW0 & Cytochrome P450 4V3 & Cyp4v3 & 0.43 & 0.42 & 1.38 & & 0.56 & \\
\hline P58710 & L-Gulonolactone oxidase & Gulo & 0.54 & 0.62 & 1.32 & & & \\
\hline Q91VS7 & Microsomal glutathione $S$-transferase 1 & Mgst1 & 0.81 & 0.81 & 1.24 & & 0.81 & \\
\hline P14211 & Calreticulin & Calr & 0.75 & 0.80 & 1.09 & & & \\
\hline $\mathrm{P} 26150$ & 3 Beta-hydroxysteroid dehydrogenase/Delta $5 \rightarrow 4$-isomerase type 3 & Hsd3b3 & 0.62 & & 1.44 & & & \\
\hline Q91WN4 & Kynurenine 3-monooxygenase & Kmo & & 0.70 & 1.29 & 0.64 & 0.70 & \\
\hline O35488 & Very long-chain acyl-CoA synthase & Slc $27 \mathrm{a} 2$ & & & 1.15 & 0.80 & 0.83 & \\
\hline Q9DCM2 & Glutathione $S$-transferase kappa 1 & Gstk1 & & & 1.62 & 0.78 & 0.72 & \\
\hline P41216 & Long-chain-fatty-acid-CoA ligase 1 & Acsl1 & & & 1.72 & 0.79 & 0.70 & \\
\hline Q9CW42 & MOSC domain-containing protein 1 , mitochondrial & Mosc1 & & & 1.41 & & 0.81 & \\
\hline
\end{tabular}

List of differentially expressed hepatic proteins with an opposite direction of changes in non-fasted HFD-fed mice and non-fasted hyperphagic mice as compared with control. The presented fold change (FC) values are relative to non-fasted control animals with adjusted $p$ value $\leq 0.05$. Bolded are gene names of eight proteins with expression level different with $\mathrm{FC}>3$ between hyperphagic and HFD-fed mice

In skeletal muscles, we found 10 consequently changed proteins in at least two groups of animals during fasting (Table S9). According to STRING, three of them indicated interactions [myosin light chain $1 / 3$ (Myl1), tropomyosin alpha-1 chain (Tpm1), and calsequestrin-1 (Casq1)]; however, these 10 proteins were not enriched in KEGG pathways. In muscle samples from 16-week-old animals, only wt mice (regardless of the diet) showed commonly changed proteins (Fig. 7c).

Next, the functional analysis of transcriptomic and proteomic data sets, differentiating non-fasting from fasting mice, were performed (Table S10). Seventeen KEGG pathways were attributed to changes in mRNA levels associated with overnight fasting. One of these pathways (Peroxisome) was selected in all groups of animals, and three others (Fatty acid metabolism; PPAR signaling; and Valine, leucine, and isoleucine degradation) were selected in at least four out of eight animal groups. Differentially expressed hepatic proteins could be assigned to nine KEGG pathways, but only in three groups of old obese mice. Of these nine pathways, five pathways (Peroxisome, Fatty acid metabolism, and three neurodegenerative diseases) were also selected upon corresponding probe sets data (Table S10). Functional analysis of muscle samples revealed 21 KEGG pathways selected from proteomic data sets and seven selected from transcriptomic data sets (Table S11). Common protein and RNA-related functional annotations were limited to only two groups of mice: young HFDfed mice and old control mice. 
Fig. 5 A protein interaction network constructed with STRING (Szklarczyk et al. 2011) for a set of 27 hepatic proteins with an opposite direction of expression in HFD-fed and hyperphagic mice when compared to control. Stronger evidences of associations are represented by thicker network edges. Node color depicts significantly enriched KEGG category assigned to a protein by STRING database in a set of 27 proteins while white nodes indicate proteins without functional annotation

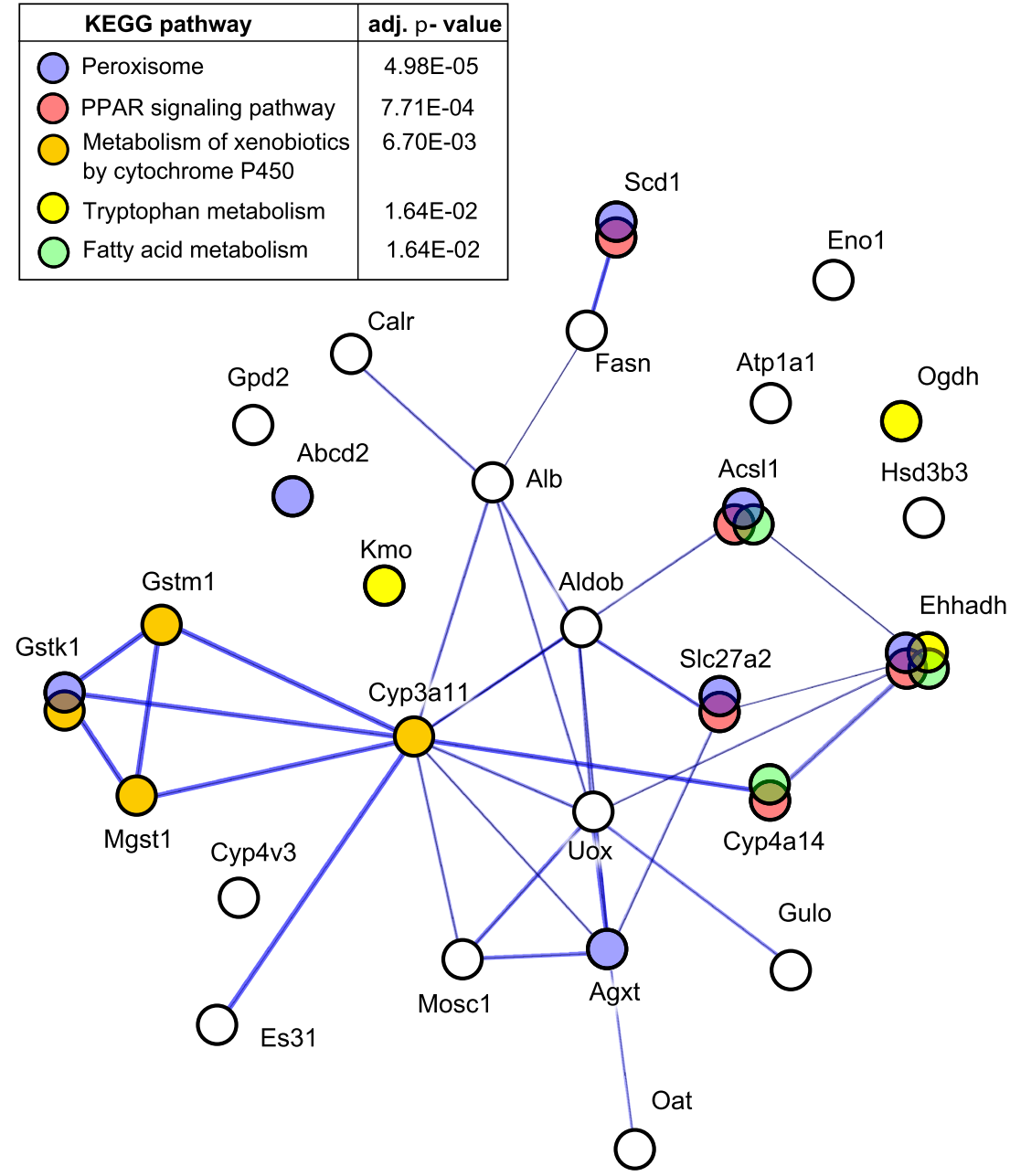

\section{Discussion}

In this study, for the first time, we demonstrate that the extent of MT proteome changes is greater in the liver than muscles in states of well-established models of obesity. The difference was captured in the number of identified proteins $-1,675$ and
704 proteins represented by at least two peptides, for liver and muscles, respectively, as well as in the number of differentially expressed proteins in these two tissues (Figs. 4 and 7). In the liver, but not in skeletal muscles, we point functional changes, depicted by KEGG pathways, distinctive to obesity (Table S7) and fasting (Table S10) conditions by collating MT proteome

Table 3 Numbers of detected proteins and numbers of proteins that corresponded to measurable mRNA used for comparisons between mice subjected to prolonged fasting or non-fasting in each group

\begin{tabular}{|c|c|c|c|c|c|c|c|c|}
\hline & \multicolumn{4}{|l|}{16 weeks } & \multicolumn{4}{|l|}{48 weeks } \\
\hline & Control & $\mathrm{ob} / \mathrm{ob}$ & $\mathrm{db} / \mathrm{db}$ & HFD & Control & $\mathrm{ob} / \mathrm{ob}$ & $\mathrm{db} / \mathrm{db}$ & HFD \\
\hline \multicolumn{9}{|l|}{ Hepatic proteins } \\
\hline $\begin{array}{l}\text { Total analyzed proteins (proteins with } \\
\text { corresponding probe sets in filtered dataset) }\end{array}$ & $1,265(961)$ & $1,249(953)$ & $1,257(957)$ & $1,249(949)$ & $1,034(794)$ & $1,096(843)$ & $1,082(829)$ & $1,056(813)$ \\
\hline Differentially expressed proteins & 51 & 31 & 23 & 22 & 28 & 21 & 16 & 21 \\
\hline \multicolumn{9}{|l|}{ Skeletal muscle proteins } \\
\hline $\begin{array}{l}\text { Total analyzed proteins (proteins with } \\
\text { corresponding probe sets in filtered dataset) }\end{array}$ & $554(428)$ & $552(423)$ & $549(425)$ & $550(423)$ & $461(355)$ & $461(353)$ & $457(350)$ & $454(349)$ \\
\hline Differentially expressed proteins & 12 & 10 & 2 & 14 & 3 & 2 & 3 & 2 \\
\hline
\end{tabular}


Fig. 6 STRING interaction network (Szklarczyk et al. 2011) for a set of 45 hepatic proteins with concordant expression during fasting in at least two groups of mice. Stronger evidences of associations are represented by thicker network edges. Node color depicts significantly enriched KEGG category assigned to a protein by STRING database in a set of 45 proteins while white nodes indicate proteins without functional annotation

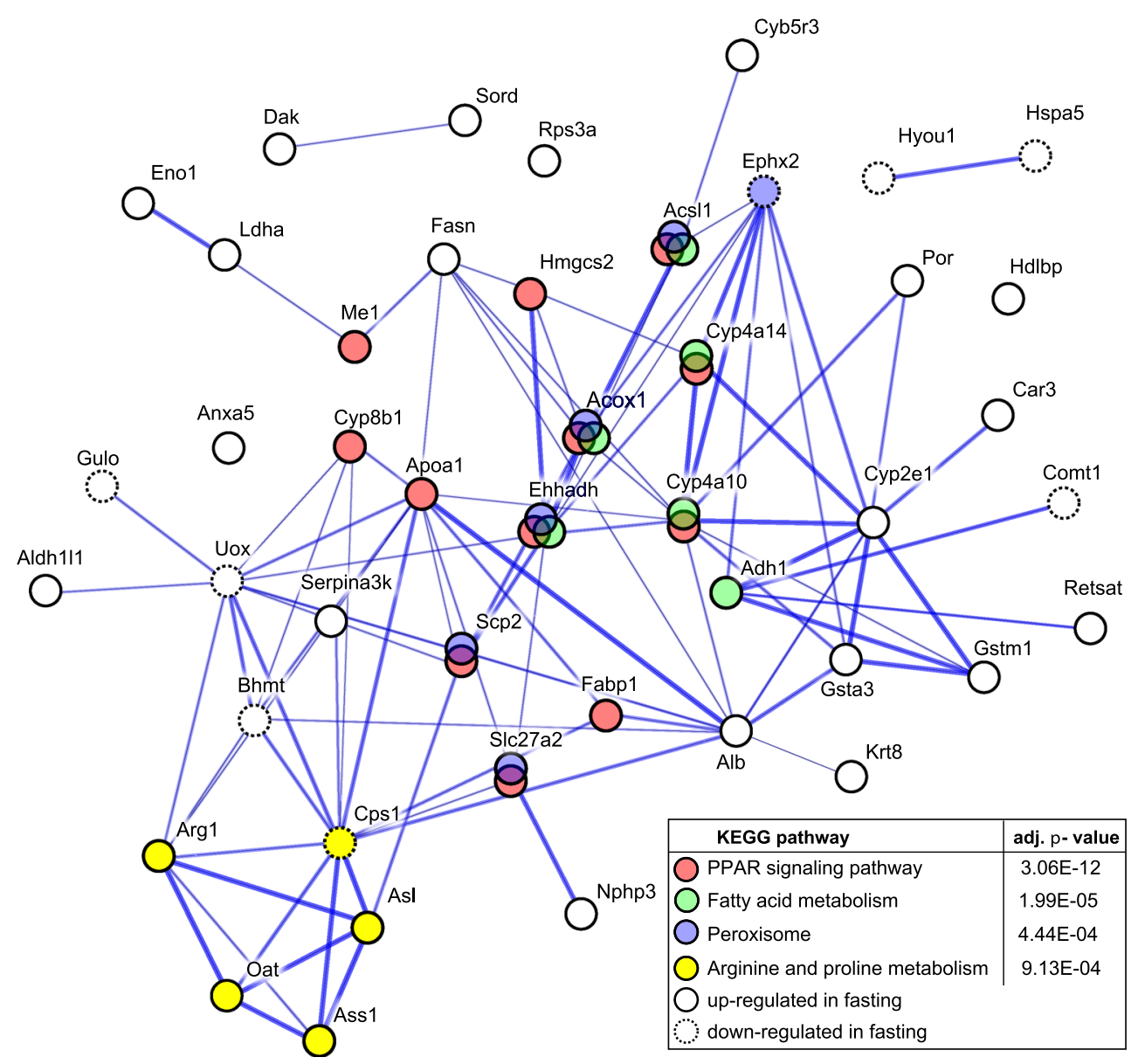

dataset with corresponding transcripts. As expected, these pathways are mostly related to fatty acids metabolism pathways.

\section{Difficulty defining the mitochondrial proteome}

Depending on the method used to identify proteins, mitochondria are thought to contain between 1,000 and 1,500 nuclearencoded proteins. However, no single method can identify all MT proteins; some are expressed at levels too low to detect, while others are expressed exclusively by specific cell types (Calvo and Mootha 2010). Furthermore, distinguishing genuine MT proteins using an MS approach may be obscured by the co-purification of protein contaminants from other membranous and MT-associated organelles (Pagliarini et al. 2008). MT proteins are directed toward the MT outer membrane either during or after translation, as their synthesis can be initiated and completed on cytoplasmic ribosomes or initiated in the cytoplasm and completed within mitochondria. Synthesis of some MT proteins takes place in the vicinity of the organelle where their corresponding mRNAs are bound to mitochondria before the initiation of translation (Pagliarini et al. 2008).
Skeletal muscle contains two types of mitochondria: intermyofibrillar mitochondria, which provide most of the energy used for muscle contraction; and subsarcolemmal mitochondria, which generate ATP for membrane functions (Ferreira et al. 2010). Most of the top ten skeletal muscle proteins identified by the greatest number of peptides in this study represented components associated with the assembly or function of vertebrate skeletal muscles, including several isoforms of myosin (Table S2). Therefore, a substantial proportion of proteins identified as non-MT might originate from organelles that are essential for MT protein translation and that colocalize topographically and/or functionally with mitochondria. Finally, some of proteins with actual MT localization might not be assigned to mitochondria in available databases (Rhee et al. 2013). With this in mind, the differentially expressed proteins and their corresponding probe sets were quantitatively analyzed and functionally annotated to pre-defined KEGG signaling pathways regardless of the established potential subcellular localization of the proteins isolated in MT fractions.

Obesity-related protein changes and functional annotations

We identified 221 hepatic and 44 muscle proteins that were expressed differently in control mice than in any obese mice 

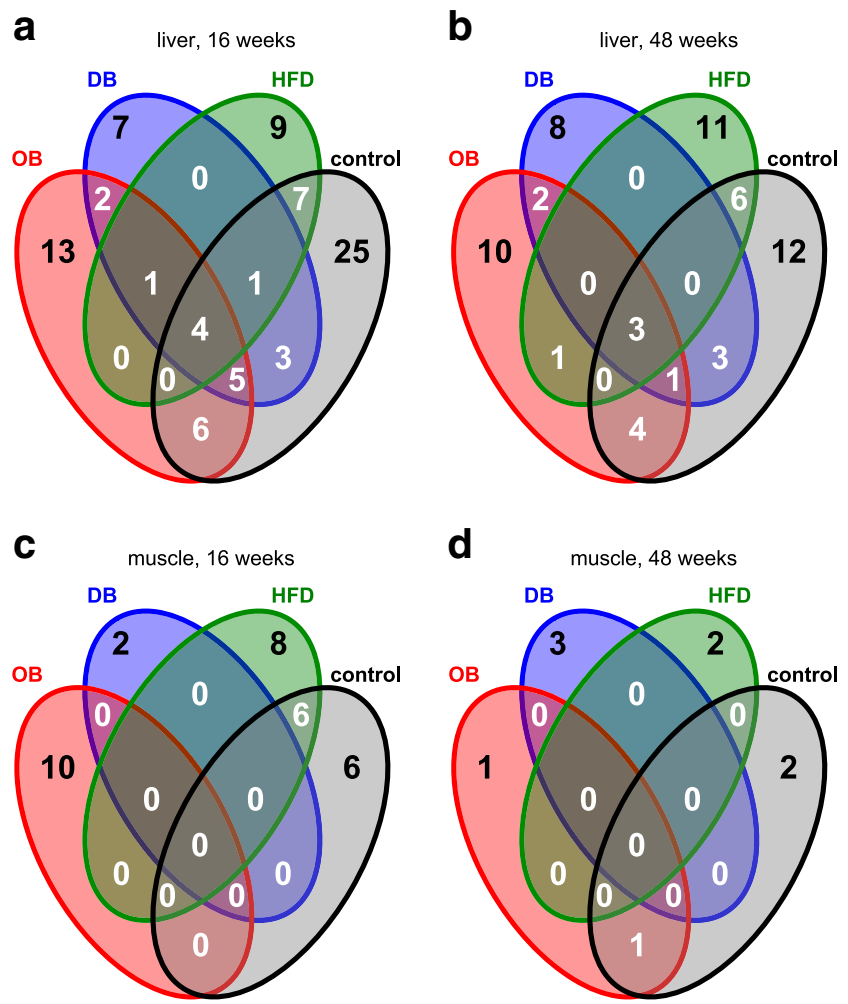

Fig. 7 Venn diagrams presenting common and unique differentially expressed proteins during overnight fasting in obese and control mice

(Tables S3 and S4). Twenty-nine of the differentially expressed hepatic proteins identified in this study were also reported as obesity-related markers in at least one of five previously published reports (Douette et al. 2005; Zhang et al. 2010; Baiges et al. 2010; Kirpich et al. 2011; Thomas et al. 2012) (Table S12). Twelve of these proteins exhibited the same direction of change as in a proteomic study by Douette et al. performed on hepatic MT fraction (Douette et al. 2005).

In the current study, proteins differentially expressed in relation to obesity, assigned to Fatty acid metabolism pathway, were upregulated in at least one obesity model and time point, and included long-chain fatty acyl-CoA synthetase (Acsm1), carnitine $O$-palmitoyltransferases (Cpt), trifunctional mitochondrial enzyme subunits $\alpha$ and $\beta$ (Hadh), medium-chain specific acyl-CoA dehydrogenase (Acadm), 3-hydroxyacyl-CoA dehydrogenase (Hsd17b10), acyl-CoA oxidase (Acox1), 3-ketoacyl-CoA thiolases (Acaa), and 3,2-trans-enoyl-CoA isomerases (Dci) (Table S3). Also, among differentially expressed proteins assigned to Glycolysis/Gluconeogenesis pathway, most were upregulated, i.e., Aldob, $\alpha$-enolase (Eno), aldehyde dehydrogenase (Aldh), L-lactate dehydrogenase (Ldha), and glyceraldehyde-3-phosphate dehydrogenase (Gapdh) (Table S3). Although for skeletal muscles, non-functional KEGG pathway was selected from either mRNA or protein data sets, several proteins participating in fatty acid or glucose metabolism processes were also upregulated (Table S4).
In ob/ob mice, the combination of glucose overproduction and enhanced fatty acid synthesis was observed, leading to further increase of insulin secretion and resistance (McGarry 1992). Consistently in our study, Fasn was also upregulated in young and old hyperphagic mice (mean $\mathrm{FC}=1.67$ ); however, it was downregulated in HFD-fed mice (mean $\mathrm{FC}=0.58$ ). Also, acetyl-CoA carboxylase 1 (Acaca) was downregulated in young mice fed with HFD, whereas different isoforms of acyl-CoA thioesterase (Acot) and Scd1, assigned to Biosynthesis of unsaturated fatty acids KEGG pathway, were upregulated in hyperphagic mice, in opposite to HFD-fed mice (Table S3).

For 27 obesity-related differentially expressed proteins, the direction of change in HFD-fed mice was opposite to that observed in hyperphagic mice (Table 2 and Fig. 5). STRING analysis reported these proteins as significantly networked and 13 of them were annotated to various metabolic KEGG pathways, with Peroxisome, PPAR signaling, and Metabolism of xenobiotics by cytochrome 450 as the most significantly enriched. Interaction between two cytochrome P450 components (Cyp3a11 and Cyp4a14) and Aldob are central elements of cluster network. Expression levels of eight (Scd1, Gstm1, Abcd2, Fasn, Cyp4V3, Es31, Oat, and Agxt) of these 27 proteins were changed by more than 3-fold when compared hyperphagic and HFD-fed mice. These differences in the direction of change could be due to specific diet composition, the later onset of adverse hepatic biochemical changes in HFD-induced obesity, or different regulation of some metabolic processes in obesity induced by hyperphagia and by HFD. Scd1 protein levels were 3.3-fold higher in hyperphagic mice than in HFD-fed mice, which is consistent with the Scd1-related model of obesity. A $S c d 1$ knock-out mutation in ob/ob mice disrupts the conversion of saturated long-chain fatty acids to monounsaturated fatty acids and significantly suppresses both obesity and hepatic steatosis (Cohen et al. 2002). The transient downregulation of $S c d 1$ induces a similar restoration of energy balance and metabolism in HFD-fed mice (Jiang et al. 2005).

Together, these results suggest that fatty acid synthesis processes might be differentially regulated in a course of obesity induced by leptin deficiency and by HFD. In opposite to HFD-fed mice, young hyperphagic mice show likely enhanced potency involved in the lipogenesis processes.

It was shown that HFD activates hepatic peroxisomal metabolism of very long-chain and branched-chain fatty acid (Kozawa et al. 2011). In Zucker rats model of obesity, peroxisomal oxidation products were shown to enter mitochondria, providing the possibility to maintain lipid disposal, even under insulin resistance condition (Noland et al. 2007). Proteomic survey in the current study confirmed the enhanced hepatic peroxisomal capacity in fatty acid oxidation. Among 221 differentially regulated proteins in liver, 24 were assigned to Peroxisome pathway and most of them were upregulated in 
16-week-old mice; six of them only in hyperphagic mice (Table S3). In old animals, differential expression of proteins involved in peroxisomal oxidative activity was not indicated, confirming that metabolic processes were altered predominantly in early phase of chronic obesity.

Combined hepatic proteomic- and transcriptomic-based functional annotations indicated that in young obese mice, alterations almost exclusively reference to metabolic processes (Table S7). Among them, mainly lipid metabolism (Fatty acid metabolism, Biosynthesis of unsaturated fatty acids, PPAR signaling, and Peroxisome) and carbohydrate metabolism (Glycolysis/Gluconeogenesis) were deregulated. Both classes of processes are essentially involved in an energy production and storage, although, comparing with other nutrient groups, fatty acids are the most efficient energy source.

Enhanced fatty acids metabolism and dysregulated gluconeogenesis were previously indicated in polygenic and dietinduced obesity mouse models, both by total hepatic gene expression analysis (Becker et al. 2004) and proteomic study of adipocyte membranes (Ho et al. 2013). Similarly, a liver MT proteins study shows coordinated upregulation of bioenergetic processes during type 2 diabetes progression in rats as potential compensation for the decreased glucose metabolism by insulin resistance (Deng et al. 2010).

Defective MT oxidation of fatty acid and the production of reactive oxygen species (ROS) may lead to the hepatic but not muscle lipid accumulation (Civitarese et al. 2007; Machado et al. 2012). Feeding with HFD for 3 days in men and for 3 weeks in mice downregulates genes necessary for muscle OXPHOS and mitochondria biogenesis (Sparks et al. 2005), and prolonged consumption of a high-caloric diet has been reported as associated with a reduction of MT capacity and respiration in mouse skeletal muscle (Bonnard et al. 2008) and rat liver (Vial et al. 2011). However, other studies on obese mice showed increased rather than decreased mitochondria density, respiration, enzyme, activity and fatty acid oxidation (Hoeks et al. 2011); expansion of muscle adipose depots in obese humans did not associate with MT dysfunction (Machado et al. 2012); and in obese rats, MT dysfunction was found to be a late event (Chanséaume et al. 2007). In accordance with these results, our study revealed that in the liver, but not in skeletal muscles, changes in MT proteins and their corresponding transcripts mostly related to fatty acids metabolism pathways.

Fasting-related protein and functional changes

An overnight fast causes enhanced metabolic stress in mice associated with a significant reduction of liver weight. This effect was particularly pronounced in lean mice in which liver mass was decreased by approximately $30 \%$ (Fig. 2b). Although not significant, a body weight reduction of approximately $15 \%$ was also noted in both lean and obese mice, similarly as reported previously (Heijboer et al. 2005). A suppressive effect of overnight fasting on serum insulin levels was also confirmed in all animals studied (not shown). However, although insulin is responsible for restraining hepatic glucose production in the fasted state and maintaining normal blood glucose concentrations (Heijboer et al. 2005), only lean (control and young HFDfed) mice exhibited decreased glucose levels (not shown).

Proteomic analysis revealed 107 proteins in liver samples and 35 proteins in muscle samples that distinguished all fasted from all non-fasted mice (Tables S8 and S9). The greatest number of overnight fasting-altered hepatic proteins was indicated in lean control mice comparing with any of obese ones (Table 3). Moreover, only few differentially expressed proteins were common between mice with obesity induced by HFD feeding and genetically, presumably indicating differences in molecular mechanisms underlining the acute fasting in both obesity models. Our study indicated 45 hepatic proteins with concordant expression change in at least two groups of animals (Fig. 6). Of these, Cyp4a14 and Cyp4a10 proteins, belonging to cytochrome $\mathrm{P} 450$ system, were consequently upregulated in all groups of animals. These proteins are encoded by wellestablished peroxisome proliferator-activated receptor (PPAR) $\alpha$-responsive genes (Patsouris et al. 2006).

PPAR signaling pathway is one of the most frequently changed in the liver during the overnight fasting, on the mRNA level (Table S10). PPARs seem to be the key regulators of overall metabolic processes in the cell as they are implicated in metabolism of lipids, carbohydrates, and proteins. PPARs are steroid nuclear receptors that function as transcription factors that mediate fatty acids regulation of gene expression, acting as the metabolic switch between fed and fasted state. There are three isoforms of PPARs: $\alpha, \beta / \delta$, and $\gamma$, with distinct tissue distributions and specific set of activated target genes (Stienstra et al. 2007).

$\operatorname{PPAR} \gamma$ is able to activate genes involved in lipogenesis in a fed state. It is abundant in adipose tissue, and plays a role in adipocyte differentiation and energy storage. Its mRNA level is increased in the adipose tissue of obese people (Vidal-Puig et al. 1997) and its activation decreased production of inflammatory cytokines in macrophages (Sharma and Staels 2007). Two other PPAR isoforms promote lipid catabolism and are found in metabolically active tissues with high fatty acids oxidation. PPAR $\alpha$ is expressed mainly in the liver, brown adipose tissue, skeletal muscle, heart, and blood cells. It is a key regulator of energy homeostasis and plays a pivotal role in the regulation of genes involved in fatty acids oxidation, metabolism of lipoproteins, gluconeogenesis, and synthesis of ketone bodies (Mandard et al. 2004). Overall, PPAR $\alpha$ activation decreased hepatic fat storage. During fasting, it decreased the expression of genes involved in the amino acids metabolism (Kersten et al. 2001). It was upregulated in mice fed with HFD (Kozawa et al. 2011). Beside metabolic processes, PPAR $\alpha$ is also involved in regulation of inflammation processes. Under activation, it 
reduces hepatic inflammation by interference with several proinflammatory transcription factors (Delerive et al. 1999), as well as by inhibition of cytokine signaling pathways via downregulation of the IL- 6 receptor (Gervois et al. 2004). PPAR $\beta / \delta$ is ubiquitously expressed and is less specific. Its functions are still the least defined, although recently its role in insulin secretion by pancreatic $\beta$-cell (Iglesias et al. 2012) and muscle regeneration (Bonala et al. 2012) have been indicated. Altogether, PPARs are among the most important processes which allow the adaptation of metabolic and functional responses to extracellular signals.

When assaying fasting regulated hepatic mRNAs and proteins to KEGG pathways, nine pathways were selected solely for old obese animals based on proteomic data; in turn, corresponding mRNAs were annotated to twice as more differentially regulated pathways in young and old mice, both lean and obese (Table S10). Such observations may suggest that transcriptional changes take place as a type of adaptive processes in response to overnight fasting. In skeletal muscles, overnight food deprivation, contrary to chronic obesity conditions, allow to point several KEGG pathways for both proteomic and corresponding transcriptomic data sets (Table S11). However, functional annotations were limited to only two groups of animals: young HFD-fed mice and old control mice.

In conclusion, we provide a comprehensive overview on the molecular, mitochondria-related changes that arise during obesity and food deprivation in two metabolically active tissues, liver and skeletal muscles. Expression levels of genes and proteins that accompanied the obesity and fasting states were quantitatively different in the two studied tissues, and both conditions induce more pronounced proteome changes in the liver than in the muscles.

Acknowledgments This work was supported by N N401 017436 grant from the Polish Ministry of Science and Higher Education and 501-1-0912-12 grant from the Medical Center for Postgraduate Education. Tymon Rubel was supported by the European Social Fund through the project "Preparation and realization of Medical Physics speciality" (UDA-POKL 04.01.01-00-0004/09-00) at the Faculty of Physics of the Warsaw University of Technology.

Open Access This article is distributed under the terms of the Creative Commons Attribution License which permits any use, distribution, and reproduction in any medium, provided the original author(s) and the source are credited.

\section{References}

Adachi J, Kumar C, Zhang Y, Mann M (2007) In-depth analysis of the adipocyte proteome by mass spectrometry and bioinformatics. Mol Cell Proteomics 6:1257-1273. doi:10.1074/mcp.M600476MCP200

Altelaar AFM, Heck AJR (2012) Trends in ultrasensitive proteomics. Curr Opin Chem Biol 16:206-213. doi:10.1016/j.cbpa.2011. 12.011
Andrikopoulos S, Blair AR, Deluca N et al (2008) Evaluating the glucose tolerance test in mice. Am J Physiol Endocrinol Metab 295:E1323E1332. doi:10.1152/ajpendo.90617.2008

Ashburner M, Ball CA, Blake JA et al (2000) Gene ontology: tool for the unification of biology. The Gene Ontology Consortium. Nat Genet 25:25-29. doi:10.1038/75556

Ayala JE, Samuel VT, Morton GJ et al (2010) Standard operating procedures for describing and performing metabolic tests of glucose homeostasis in mice. Dis Model Mech 3:525-534. doi:10.1242/ dmm.006239

Baiges I, Palmfeldt J, Bladé C et al (2010) Lipogenesis is decreased by grape seed proanthocyanidins according to liver proteomics of rats fed a high fat diet. Mol Cell Proteomics 9:1499-1513. doi:10.1074/ mcp.M000055-MCP201

Becker W, Kluge R, Kantner T et al (2004) Differential hepatic gene expression in a polygenic mouse model with insulin resistance and hyperglycemia: evidence for a combined transcriptional dysregulation of gluconeogenesis and fatty acid synthesis. J Mol Endocrinol $32: 195-208$

Boirie Y (2003) Insulin regulation of mitochondrial proteins and oxidative phosphorylation in human muscle. Trends Endocrinol Metab 14:393-394

Bonala S, Lokireddy S, Arigela $\mathrm{H}$ et al (2012) Peroxisome proliferatoractivated receptor $\beta / \delta$ induces myogenesis by modulating myostatin activity. J Biol Chem 287:12935-12951. doi:10.1074/jbc.M111. 319145

Bonnard C, Durand A, Peyrol S et al (2008) Mitochondrial dysfunction results from oxidative stress in the skeletal muscle of diet-induced insulin-resistant mice. J Clin Invest 118:789-800. doi:10.1172/ JCI32601

Calvo SE, Mootha VK (2010) The mitochondrial proteome and human disease. Annu Rev Genomics Hum Genet 11:25-44. doi:10.1146/ annurev-genom-082509-141720

Chanséaume E, Tardy A-L, Salles J et al (2007) Chronological approach of diet-induced alterations in muscle mitochondrial functions in rats. Obesity (Silver Spring) 15:50-59. doi:10.1038/oby. 2007.511

Civitarese AE, Smith SR, Ravussin E (2007) Diet, energy metabolism and mitochondrial biogenesis. Curr Opin Clin Nutr Metab Care 10: 679-687. doi:10.1097/MCO.0b013e3282f0ecd2

Cohen P, Miyazaki M, Socci ND et al (2002) Role for stearoyl-CoA desaturase-1 in leptin-mediated weight loss. Science 297:240-243. doi:10.1126/science. 1071527

De Wilde J, Mohren R, van den Berg S et al (2008) Short-term high fatfeeding results in morphological and metabolic adaptations in the skeletal muscle of C57BL/6J mice. Physiol Genomics 32:360-369. doi:10.1152/physiolgenomics.00219.2007

De Wilde J, Smit E, Mohren R et al (2009) An 8-week high-fat diet induces obesity and insulin resistance with small changes in the muscle transcriptome of $\mathrm{C} 57 \mathrm{BL} / 6 \mathrm{~J}$ mice. J Nutrigenet Nutrigenomics 2:280-291. doi:10.1159/000308466

Delerive P, De Bosscher K, Besnard S et al (1999) Peroxisome proliferator-activated receptor alpha negatively regulates the vascular inflammatory gene response by negative cross-talk with transcription factors NF-kappaB and AP-1. J Biol Chem 274:3204832054

Deng W-J, Nie S, Dai J et al (2010) Proteome, phosphoproteome, and hydroxyproteome of liver mitochondria in diabetic rats at early pathogenic stages. Mol Cell Proteomics 9:100-116. doi:10.1074/ mcp.M900020-MCP200

Development Core Team R (2011) R: a language and environment for statistical. Computing 1:409. doi:10.1007/978-3-540-74686-7

Douette P, Navet R, Gerkens P et al (2005) Steatosis-induced proteomic changes in liver mitochondria evidenced by two-dimensional differential in-gel electrophoresis. J Proteome Res 4:2024-2031. doi:10. $1021 / \mathrm{pr} 050187 \mathrm{z}$ 
Du P, Kibbe WA, Lin SM (2008) lumi: a pipeline for processing Illumina microarray. Bioinformatics 24:1547-1548. doi:10.1093/ bioinformatics/btn224

Eccleston HB, Andringa KK, Betancourt AM, et al (2011) Chronic exposure to a high-fat diet induces hepatic steatosis, impairs nitric oxide bioavailability, and modifies the mitochondrial proteome in mice. Antioxid Redox Signal 15:447-459. doi:10.1089/ars.2010.3395

Ferreira R, Vitorino R, Alves RMP et al (2010) Subsarcolemmal and intermyofibrillar mitochondria proteome differences disclose functional specializations in skeletal muscle. Proteomics 10:3142-3154. doi:10.1002/pmic.201000173

Gentleman RC, Carey VJ, Bates DM et al (2004) Bioconductor: open software development for computational biology and bioinformatics. Genome Biol 5:R80. doi:10.1186/gb-2004-5-10-r80

Gervois P, Kleemann R, Pilon A et al (2004) Global suppression of IL-6induced acute phase response gene expression after chronic in vivo treatment with the peroxisome proliferator-activated receptor-alpha activator fenofibrate. J Biol Chem 279:16154-16160. doi:10.1074/ jbc.M400346200

Greco D, Kotronen A, Westerbacka J et al (2008) Gene expression in human NAFLD. Am J Physiol Gastrointest Liver Physiol 294: G1281-G1287. doi:10.1152/ajpgi.00074.2008

Heijboer AC, Donga E, Voshol PJ et al (2005) Sixteen hours of fasting differentially affects hepatic and muscle insulin sensitivity in mice. J Lipid Res 46:582-588. doi:10.1194/jlr.M400440-JLR200

Ho JH, Lee OK, Fu Y-J et al (2013) An iTRAQ proteomic study reveals an association between diet-induced enhanced fatty acid metabolism and the development of glucose intolerance in prediabetic mice. $\mathrm{J}$ Proteome Res 12:1120-1133. doi:10.1021/pr300662j

Hoeks J, de Wilde J, Hulshof MFM et al (2011) High fat diet-induced changes in mouse muscle mitochondrial phospholipids do not impair mitochondrial respiration despite insulin resistance. PLoS ONE 6:e27274. doi:10.1371/journal.pone.0027274

Iglesias J, Barg S, Vallois D et al (2012) PPAR $\beta / \delta$ affects pancreatic $\beta$ cell mass and insulin secretion in mice. J Clin Invest 122:41054117. doi:10.1172/JCI42127

Jiang G, Li Z, Liu F et al (2005) Prevention of obesity in mice by antisense oligonucleotide inhibitors of stearoyl-CoA desaturase-1. J Clin Invest 115:1030-1038. doi:10.1172/JCI23962

Kanehisa M, Goto S (2000) KEGG: Kyoto encyclopedia of genes and genomes. Nucleic Acids Res 28:27-30

Kapoor KN, Barry DT, Rees RC et al (2009) Estimation of peptide concentration by a modified bicinchoninic acid assay. Anal Biochem 393:138-140. doi:10.1016/j.ab.2009.06.016

Kennedy AJ, Ellacott KLJ, King VL, Hasty AH (2010) Mouse models of the metabolic syndrome. Dis Model Mech 3:156-166. doi:10.1242/ dmm.003467

Kersten S, Mandard S, Escher P et al (2001) The peroxisome proliferatoractivated receptor alpha regulates amino acid metabolism. FASEB J 15:1971-1978. doi:10.1096/fj.01-0147com

Kim E (2010) Insulin resistance at the crossroads of metabolic syndrome: systemic analysis using microarrays. Biotechnol J 5:919-929. doi: 10.1002/biot.201000048

Kirpich IA, Gobejishvili LN, Bon Homme M et al (2011) Integrated hepatic transcriptome and proteome analysis of mice with high-fat diet-induced nonalcoholic fatty liver disease. J Nutr Biochem 22: 38-45. doi:10.1016/j.jnutbio.2009.11.009

Kozawa S, Honda A, Kajiwara N et al (2011) Induction of peroxisomal lipid metabolism in mice fed a high-fat diet. Mol Med Rep 4:11571162. doi: $10.3892 / \mathrm{mmr} .2011 .560$

Larter CZ, Yeh MM (2008) Animal models of NASH: getting both pathology and metabolic context right. J Gastroenterol Hepatol 23: 1635-1648. doi:10.1111/j.1440-1746.2008.05543.x

Luo M, Mengos AE, Stubblefield TM, Mandarino LJ (2012) High fat diet-induced changes in hepatic protein abundance in mice. J Proteomics Bioinform 05:60-66. doi:10.4172/jpb.1000214
Machado MV, Ferreira DMS, Castro RE et al (2012) Liver and muscle in morbid obesity: the interplay of fatty liver and insulin resistance. PLoS ONE 7:e31738. doi:10.1371/journal.pone.0031738

Malinowska A, Kistowski M, Bakun M et al (2012) Diffprot-software for non-parametric statistical analysis of differential proteomics data. J Proteomics 75:4062-4073. doi:10.1016/j.jprot.2012.05.030

Mandard S, Müller M, Kersten S (2004) Peroxisome proliferatoractivated receptor alpha target genes. Cell Mol Life Sci 61:393416. doi:10.1007/s00018-003-3216-3

McGarry JD (1992) What if Minkowski had been ageusic? An alternative angle on diabetes. Science 258:766-770

Mikula M, Dzwonek A, Hennig EE, Ostrowski J (2005) Increased mitochondrial gene expression during L6 cell myogenesis is accelerated by insulin. Int J Biochem Cell Biol 37:1815-1828. doi:10. 1016/j.biocel.2005.02.008

Mikula M, Dzwonek A, Karczmarski J et al (2006) Landscape of the hnRNP K protein-protein interactome. Proteomics 6:2395-2406. doi:10.1002/pmic.200500632

Mikula M, Rubel T, Karczmarski J et al (2011) Integrating proteomic and transcriptomic high-throughput surveys for search of new biomarkers of colon tumors. Funct Integr Genomics 11:215-224. doi: 10.1007/s10142-010-0200-5

Naik A, Košir R, Rozman D (2013) Genomic aspects of NAFLD pathogenesis. Genomics 102:84-95. doi:10.1016/j.ygeno.2013.03.007

Noland RC, Woodlief TL, Whitfield BR et al (2007) Peroxisomal-mitochondrial oxidation in a rodent model of obesity-associated insulin resistance. Am J Physiol Endocrinol Metab 293:E986-E1001. doi: 10.1152/ajpendo.00399.2006

Ostrowski J, Klimek-Tomczak K, Wyrwicz LS et al (2004) Heterogeneous nuclear ribonucleoprotein $\mathrm{K}$ enhances insulin-induced expression of mitochondrial UCP2 protein. J Biol Chem 279:5459954609. doi:10.1074/jbc.M406753200

Pagliarini DJ, Calvo SE, Chang B et al (2008) A mitochondrial protein compendium elucidates complex I disease biology. Cell 134:112123. doi:10.1016/j.cell.2008.06.016

Panchal SK, Brown L (2011) Rodent models for metabolic syndrome research. J Biomed Biotechnol 2011:351982. doi:10.1155/2011/351982

Park J-E, Kim HT, Lee S, et al (2011) Differential expression of intermediate filaments in the process of developing hepatic steatosis. Proteomics 11:2777-2789. doi:10.1002/pmic.201000544

Patsouris D, Reddy JK, Müller M, Kersten S (2006) Peroxisome proliferator-activated receptor alpha mediates the effects of highfat diet on hepatic gene expression. Endocrinology 147:1508-1516. doi:10.1210/en.2005-1132

Rhee H-W, Zou P, Udeshi ND et al (2013) Proteomic mapping of mitochondria in living cells via spatially restricted enzymatic tagging. Science 339:1328-1331. doi:10.1126/science.1230593

Samuel VT, Shulman GI (2012) Mechanisms for insulin resistance: common threads and missing links. Cell 148:852-871. doi:10. 1016/j.cell.2012.02.017

Sharma AM, Staels B (2007) Review: peroxisome proliferator-activated receptor gamma and adipose tissue - understanding obesity-related changes in regulation of lipid and glucose metabolism. J Clin Endocrinol Metab 92:386-395. doi:10.1210/jc.2006-1268

Sharma MR, Polavarapu R, Roseman D et al (2006) Transcriptional networks in a rat model for nonalcoholic fatty liver disease: a microarray analysis. Exp Mol Pathol 81:202-210. doi:10.1016/j. yexmp.2006.06.001

Sharma A, Bartell SM, Baile CA et al (2010) Hepatic gene expression profiling reveals key pathways involved in leptin-mediated weight loss in ob/ob mice. PLoS ONE 5:e12147. doi:10.1371/journal.pone.0012147

Sparks LM, Xie H, Koza RA et al (2005) A high-fat diet coordinately downregulates genes required for mitochondrial oxidative phosphorylation in skeletal muscle. Diabetes 54:1926-1933

Stienstra R, Duval C, Müller M, Kersten S (2007) PPARs, obesity, and inflammation. PPAR Res 2007:95974. doi:10.1155/2007/95974 
Sun G (2007) Application of DNA microarrays in the study of human obesity and type 2 diabetes. OMICS 11:25-40. doi:10.1089/omi. 2006.0003

Szklarczyk D, Franceschini A, Kuhn M et al (2011) The STRING database in 2011: functional interaction networks of proteins, globally integrated and scored. Nucleic Acids Res 39:D561-D568. doi: 10.1093/nar/gkq973

Thomas A, Stevens AP, Klein MS et al (2012) Early changes in the liversoluble proteome from mice fed a nonalcoholic steatohepatitis inducing diet. Proteomics 12:1437-1451. doi:10.1002/pmic. 201100628
Vial G, Dubouchaud H, Couturier K et al (2011) Effects of a high-fat diet on energy metabolism and ROS production in rat liver. J Hepatol 54: 348-356. doi:10.1016/j.jhep.2010.06.044

Vidal-Puig AJ, Considine RV, Jimenez-Liñan M et al (1997) Peroxisome proliferator-activated receptor gene expression in human tissues. Effects of obesity, weight loss, and regulation by insulin and glucocorticoids. J Clin Invest 99:2416-2422. doi:10.1172/JCI119424

Zhang X, Yang J, Guo Y et al (2010) Functional proteomic analysis of nonalcoholic fatty liver disease in rat models: enoyl-coenzyme a hydratase down-regulation exacerbates hepatic steatosis. Hepatology 51:1190-1199. doi:10.1002/hep.23486 